\title{
The human long non-coding RNA-RoR is a p53 repressor in response to DNA damage
}

\author{
Ali Zhang ${ }^{1, *}$, Nanjiang Zhou ${ }^{1, *}$, Jianguo Huang ${ }^{1, *}$, Qian Liu ${ }^{1}$, Koji Fukuda ${ }^{1}$, Ding Ma ${ }^{2}$, Zhaohui Lu ${ }^{3}$, \\ Cunxue $\mathrm{Bai}^{4}$, Kounosuke Watabe ${ }^{1}$, Yin-Yuan $\mathrm{Mo}^{1,5}$ \\ ${ }^{I}$ Department of Medical Microbiology, Immunology and Cell Biology, Southern Illinois University School of Medicine, Springfield, \\ IL 62794-9621, USA; ${ }^{2}$ Cancer Biology Research Center, Tongji Hospital, Tongji Medical College, Huazhong University of Science \\ and Technology, Wuhan, Hubei 430030, China; ${ }^{3}$ Department of Endocrinology, PLA General Hospital, Beijing 100853, China; \\ ${ }^{4}$ Department of Pulmonary Medicine, Zhongshan Hospital, Fudan University, Shanghai 200032, China: ${ }^{5}$ Cancer Institute, Univer- \\ sity of Mississippi Medical Center Cancer Institute, 2500 N State St, Guyton 2, Suite G651, Jackson, MS 39216-4505, USA
}

It is well known that upon stress, the level of the tumor suppressor p53 is remarkably elevated. However, despite extensive studies, the underlying mechanism involving important inter-players for stress-induced p53 regulation is still not fully understood. We present evidence that the human lincRNA-RoR (RoR) is a strong negative regulator of p53. Unlike MDM2 that causes p53 degradation through the ubiquitin-proteasome pathway, RoR suppresses p53 translation through direct interaction with the heterogeneous nuclear ribonucleoprotein I (hnRNP I). Importantly, a 28-base RoR sequence carrying hnRNP I binding motifs is essential and sufficient for p53 repression. We further show that RoR inhibits p53-mediated cell cycle arrest and apoptosis. Finally, we demonstrate a RoR-p53 autoregulatory feedback loop where $\mathrm{p} 53$ transcriptionally induces RoR expression. Together, these results suggest that the RoRhnRNP I-p53 axis may constitute an additional surveillance network for the cell to better respond to various stresses. Keywords: IncRNA; p53; hnRNPI

Cell Research (2013) 23:340-350. doi:10.1038/cr.2012.164; published online 4 December 2012

\section{Introduction}

The tumor suppressor $\mathrm{p} 53$ is a master gene regulator controlling diverse cellular processes including DNA repair, cell cycle progression, differentiation, cell growth, senescence and apoptosis [1,2]. Because of its importance, $\mathrm{p} 53$ expression is regulated by a variety of means, such as transcriptional and translational control, alternative splicing, posttranslational modifications and regulation of subcellular localization. Thus, in the unstressed cells, the p53 level remains low; however, upon stress, the p53 level is increased significantly. Although it is believed that such an increase in the p53 level is mainly due to increased stability through posttranslational modi-

\footnotetext{
*These three authors contributed equally to this work.

Correspondence: Yin-Yuan Mo

Tel: +1-601-815-6849

E-mail: ymo@umc.edu

Received 20 August 2012; revised 24 September 2012; accepted 25 October 2012; published online 4 December 2012
}

fications such as phosphorylation [3], recent evidence indicates that $\mathrm{p} 53$ translational regulation after DNA damage may also play an important role [4].

Protein-coding genes account for only about $2 \%$ of the human genome, whereas the vast majority of transcripts consist of the non-coding RNAs, among which are long non-coding RNAs (lncRNAs) with a molecular weight of over 200 bases in length [5]. Emerging evidence indicates that lncRNAs, like protein-coding genes, are transcribed mainly by RNA polymerase II; they are spliced products via canonical genomic splice site motifs, frequently ended with a poly A tail. Moreover, they are often regulated by well-established transcription factors and are expressed in a tissue-specific manner [6]. A variety of previously identified RNA species belong to this group, including antisense RNAs, transcribed ultraconserved regions and pseudogenes. For example, antisense RNAs are transcribed from the opposite strand of a protein-coding gene and frequently overlap the corresponding gene [7]; pseudogenes such as PTENP1 function as a microRNA decoy [8]. Overexpression of the PTENP1 
leads to an increased level of PTEN and causes cellular growth inhibition, an effect dependent on the presence of mature miRNAs [9]. However, the most important group of lncRNAs are probably long or large intergenic non-coding RNAs (lincRNAs). Despite relatively newly identified, they have been shown to play a critical role in regulation of a variety of cellular functions and disease progressions including stem cell state and cancer metastasis [10-16]. This may have to do with their ability to regulate gene expression. In this regard, the proposed functions for lincRNAs include as signals for transcription; as decoys to titrate transcription factors; as guides so that chromatin-modifying enzymes can be recruited to target genes; and as scaffolds to bring together multiple proteins to form ribonucleoprotein complexes $[17,18]$.

The human lincRNA-RoR (RoR) is a recently identified lincRNA that is capable of reprogramming differentiated cells to induced pluripotent stem cells (iPSCs) [19]. While knockdown of RoR decreases, overexpression increases the number of iPSC colonies formed without affecting cell number. The RoR gene is $2.6 \mathrm{~kb}$ in length, located in chromosome 18 (hg19 chr18:54,721,802$54,739,350)$, consisting of four exons. RoR is highly expressed in embryonic stem cells and iPSCs, which is likely due to the regulation of RoR by pluripotency transcription factors such as Oct4, Sox 2 and Nanog. Of interest, knockdown of RoR leads to a modest increase in apoptosis and activation of p53 pathways [19]. However, the underlying mechanisms remain to be determined. In particular, it is unknown whether RoR plays any role in p53 regulation at the translational level.

In this study, we show that RoR functions as a negative regulator of $\mathrm{p} 53$ through interaction with an RNA binding protein, heterogeneous nuclear ribonucleoprotein I (hnRNP I). While RoR has little effect on p53 in the unstressed cells, it significantly suppresses the DNA damage-induced p53 through a translation repression mechanism. As a result, RoR modulates the p53-regulated cellular processes such as cell cycle progression and apoptosis, and the expression of p53 downstream target genes. Furthermore, RoR and p53 form an autoregulatory feedback loop where p53 positively regulates RoR expression by direct interaction with a 553 response element in the putative RoR promoter.

\section{Results}

\section{RoR suppresses the DNA damage-induced p53}

Since a previous study showed that knockdown of p53 can partially rescue the apoptotic phenotype caused by ablation of RoR [19], this prompted us to investigate the role of RoR in $\mathrm{p} 53$ expression and the $\mathrm{p} 53$ regulatory network. Although RoR had little effect on p53 in the unstressed cells, it remarkably suppressed the doxorubicin (doxo)-induced p53 (Figure 1A). A large increase of p53 level was seen in the doxo-treated MCF-7 cells compared to no doxo control cells; however, the same cells transfected with RoR revealed a much lower level of p53 induction than vector control. This was also seen in HCT-
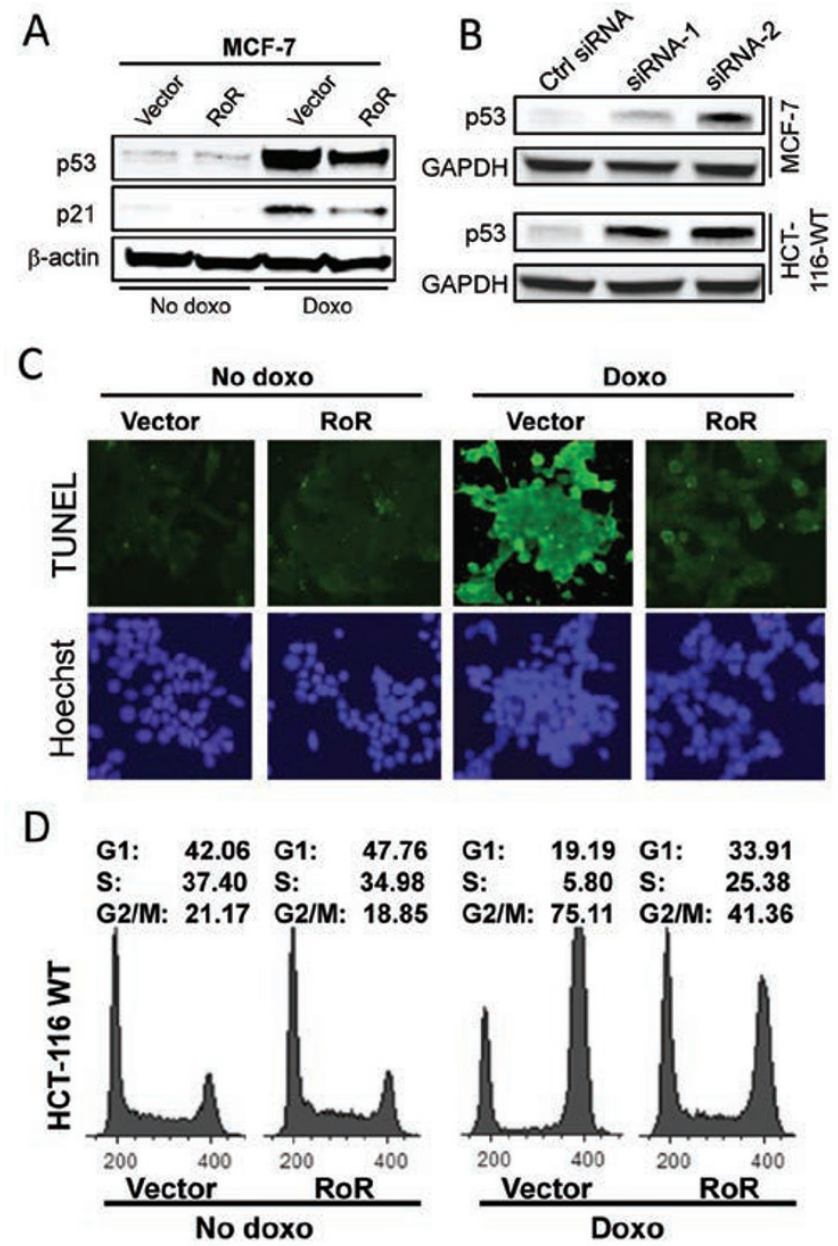

Figure 1 RoR suppresses the doxo-induced p53 and the p53mediated cellular function. (A) Detection of p53 in MCF-7 cells with or without doxo. Cells were transfected with vector or RoR and then treated with doxo at $1 \mu \mathrm{g} / \mathrm{ml}$ for $24 \mathrm{~h}$. Total cellular protein was then extracted for western blot. (B) Suppression of RoR by RNAi induces p53. MCF-7 and HCT-116 WT cells were transfected with control siRNA or RoR-siRNA-1 or RoR-siRNA-2. (C) RoR inhibits the doxo-induced apoptosis. The transfected MCF-7 cells were treated with doxo at $2 \mu \mathrm{g} / \mathrm{ml}$ for $24 \mathrm{~h}$ before TUNEL assay was performed. A high concentration of doxo was used to detect cell apoptosis. (D) RoR suppresses the doxo-induced G2/M arrest. HCT-116 WT cells were transfected with vector or RoR, and then treated with doxo at $0.1 \mu \mathrm{g} / \mathrm{ml}$ for $24 \mathrm{~h}$, followed by FACS cell cycle analysis. A low concentration of doxo was used to detect cell cycle arrest. 


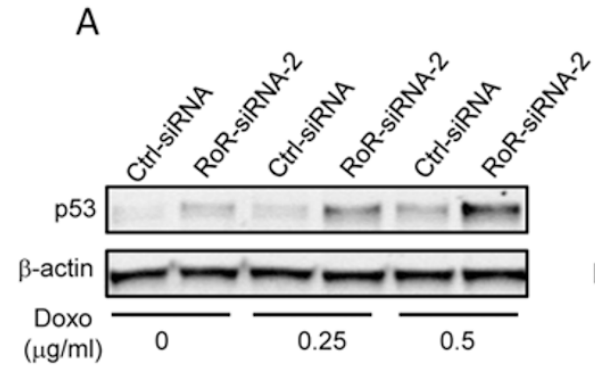

D

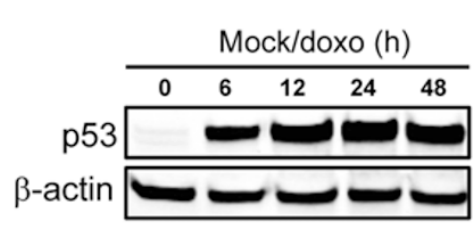

No $\mathrm{CHX}$
$E$

\begin{tabular}{ccc}
\multicolumn{3}{c}{ Ctrl/doxo (h) } \\
\hline $6 \quad 12 \quad 24 \quad 48$ \\
\hline
\end{tabular}

$\mathrm{CHX}$

\section{$\mathrm{F}$}

B

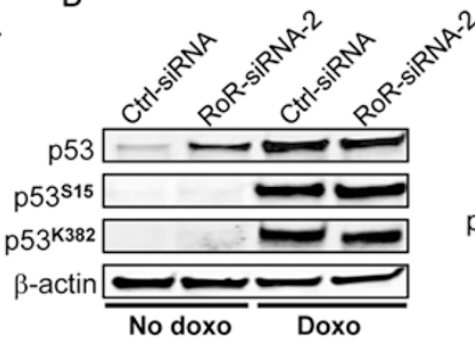

$\beta$-actin

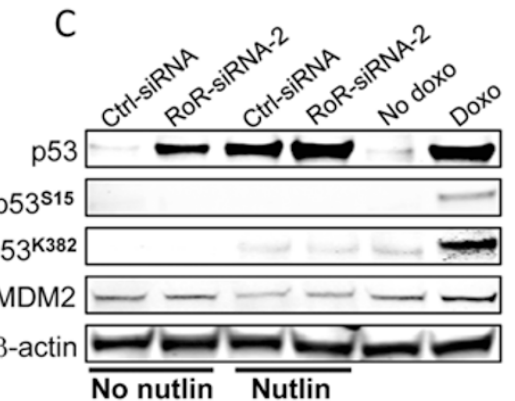

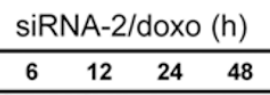

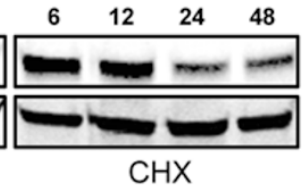

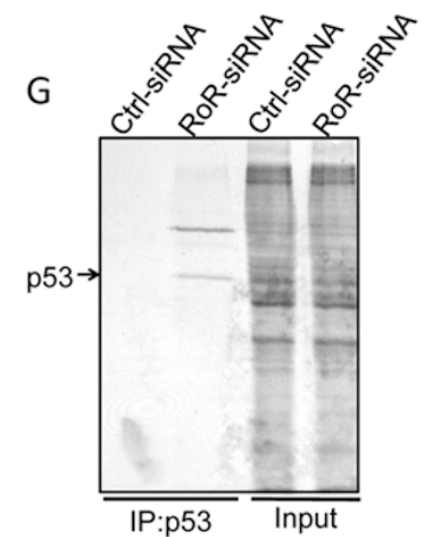

Figure 2 Translational repression of p53 by RoR. (A) Effect of RoR-siRNA on the p53 level with or without doxo. MCF-7 cells were first transfected with control or RoR-siRNA-2 overnight and then treated with doxo at the indicated concentrations for $16 \mathrm{~h}$ before harvesting for western blot. (B) RoR-siRNA induces p53 without causing p53 phosphorylation or acetylation. For doxo treatment, MCF-7 cells were transfected with control siRNA or RoR-siRNA-2 overnight and then treated with doxo at $0.5 \mu \mathrm{g} / \mathrm{ml}$ for $24 \mathrm{~h}$. (C) Effect of RoR-siRNA combined with nutlin on p53. MCF-7 cells were transfected with control siRNA or RoR-siRNA-2 and then treated with nutlin at $0.5 \mu \mathrm{g} / \mathrm{ml}$ for $24 \mathrm{~h}$ before the cells were harvested for western blot. (D-F) RoRsiRNA does not change the stability of the doxo-induced p53. MCF-7 cells were transfected with control siRNA or RoR-siRNA-2 and then treated with $\mathrm{CHX}$ at $20 \mu \mathrm{g} / \mathrm{ml}$ for $30 \mathrm{~min}$, followed by doxo treatment at $0.5 \mu \mathrm{g} / \mathrm{ml}$. The cells were harvested for western blot at the indicated time points after doxo treatment. (G) RoR-siRNA increases the newly synthesized p53 level as detected by pulse-chase experiments. MCF-7 cells were first transfected with control siRNA or RoR-siRNA-2 overnight and newly synthesized protein was labeled with ${ }^{35} \mathrm{~S}$ methionine, followed by IP with p53 antibody.

116 cells expressing wild-type p53 (HCT-116-WT) but not in p53-null cells (HCT-116-p53 null) (Supplementary information, Figure S1A). Consistent with this finding, p53 downstream genes p21 and miR-145 [20] were also suppressed (Figure 1A; Supplementary information, Figure S1B). Similarly, RoR was able to suppress the ultraviolet C (UVC)-induced p53 (Supplementary information, Figure S1C). In contrast, RoR-siRNAs induced p53 (Figure 1B; Supplementary information, Figure S1D). Given that p53 is a key factor regulating apoptosis and cell cycle progression, we first determined whether RoR affects p53-mediated apoptosis. Consistent with the suppression of $\mathrm{p} 53$, RoR reduced the $\mathrm{p} 53$-mediated apoptosis, as detected by TUNEL (Terminal deoxynucleotidyl transferase dUTP nick end labeling) assays (Figure 1C). Similarly, RoR reduced the p53-mediated G2/M arrest (Figure 1D). In the absence of doxo, RoR had little ef- fect on cell cycle profile in HCT-116-WT cells. However, RoR remarkably reduced the doxo-induced G2/M population, from $75.1 \%$ down to $41.4 \%$ (Figure 1D). On the other hand, such negative effect on G2/M population was not seen in the p53-null cells (Supplementary information, Figure S2).

RoR or RoR-siRNA did not change p53 mRNA levels in the presence or absence of doxo (Supplementary information, Figure S3A and S3B), suggesting that a posttranscriptional regulation mechanism was involved. It is known that DNA damage can induce p53 by increasing its stability (e.g., protein phosphorylation) and/or stimulating its translation [4]. We found that RoR-siRNA alone induced p53 (Figures 2A and 1B) and after treatment with the low concentration of doxo $(0.2 \mu \mathrm{g} / \mathrm{ml})$ for $16 \mathrm{~h}$, RoRsiRNA further induced p53 (Figure 2A), suggesting that the endogenous RoR could regulate the p53 level during 
DNA damage. Moreover, although RoR-siRNA alone was able to increase the p53 level, it did not cause p53 phosphorylation or acetylation (Figure 2B), both of which are believed to contribute to the p53 stability or activity [21, 22]. Nutlin is a non-genotoxic agent that induces p53 by directly targeting MDM2 [23], a well-characterized negative regulator of $\mathrm{p} 53$. As expected, nutlin induced $\mathrm{p} 53$, but did not cause phosphorylation or acetylation (Figure 2C). These results suggest that RoR-siRNA-mediated p53 induction may not be due to phosphorylation or acetylation. To further determine the effect of suppression of RoR on the p53 stability, we first transfected cells with RoRsiRNA and then treated them with the protein synthesis inhibitor cycloheximide (CHX) for $30 \mathrm{~min}$, followed by doxo treatment. It is evident that $\mathrm{CHX}$ reduced the doxoinduced p53 level over the time (Figure 2D vs 2E-2F); however, the $\mathrm{p} 53$ protein stability was about the same between control siRNA and RoR-siRNA (Figure 2E and $2 \mathrm{~F})$. Finally, pulse-chase experiment with ${ }^{35} \mathrm{~S}$ methionine showed a newly synthesized p53 band in the RoR-siRNA transfected cells, whereas no such a protein band was detected in the control siRNA transfected cells (Figure 2G). Therefore, at least translational repression contributes to
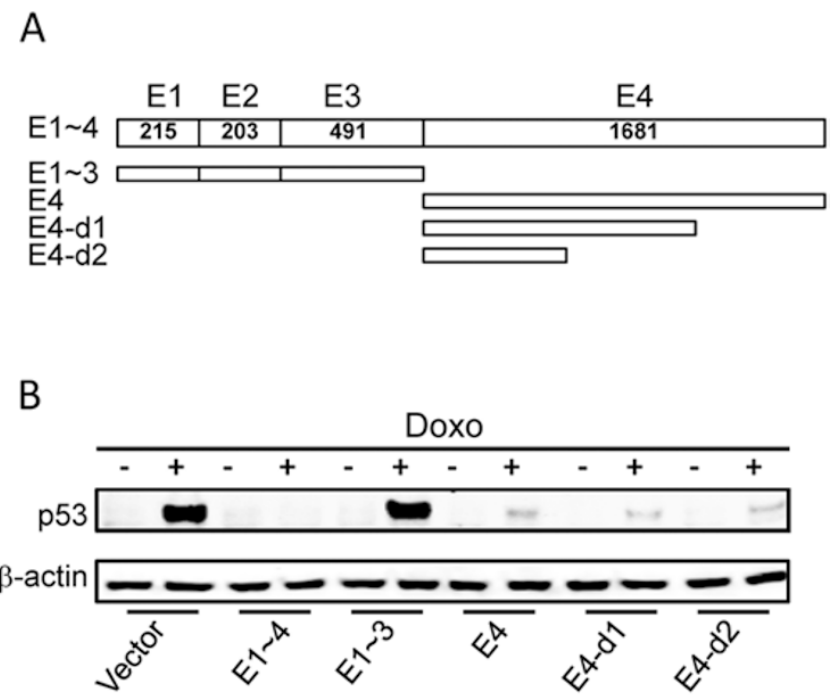

Figure 3 Identification of the suppressive activity of RoR within exon 4 (E4). (A) Schematic description of RoR deletion constructs. RoR consists of 4 exons. A series of deletions were generated using standard PCR approaches to define which region is responsible for p53 repression. E1 stands for exon 1 and so on. (B) Effect of RoR deletions on p53 repression as detected by western blot. MCF-7 cells were transfected with vector alone or full-length RoR (E1 4) or deletion constructs of RoR and then treated with doxo at $1 \mu \mathrm{g} / \mathrm{ml}$ for $24 \mathrm{~h}$ before western blot. the RoR-mediated suppression of $\mathrm{p} 53$.

\section{RoR directly interacts with hnRNP I}

RoR consists of 4 exons with the largest exon 4 of $>$ 1500 bp (Figure 3A). Of interest, DNA sequence homology search with BLAST program identified about $95 \%$ identity to the counterpart of chimpanzee sequence. However, UCSC genome browser found no homolog in mouse, dog and others except Rhesus (Supplementary information, Figure S4), suggesting that RoR is poorly conserved at the primary sequences, even though lncRNAs could be relatively conserved in terms of RNA secondary structures [24]. Deletion analysis combined with western blot identified an active region within exon 4 (Figure 3B). Further deletion analysis defined a $\sim 500 \mathrm{bp}$ fragment (E4-d2) capable of suppressing p53. Therefore, E4-d2 was used in the RNA precipitation experiments (Supplementary information, Figure S5) to identify RoR binding partner(s).

After mixing the biotin-labeled probes with total cellular extract and then separation in SDS-PAGE, silver staining of the gel revealed a doublet band ( $\sim 59 \mathrm{kDa})$ specific to the E4-d2 probe (Figure 4A; Supplementary information, Figure S6A). Mass spectrometry analysis indicated that this doublet band is hnRNP I (also called PTBP1) (Supplementary information, Figure S6B). Western blot confirmed the nature of the protein band (Figure 4B; Supplementary information, Figure S7A). However, we found no evidence of RoR interacting with MDM2 or other well-known negative regulators including MDMX [25], COP1 [26], Pirh2 [27] or p53 translation regulators including RPL26 $[28,29]$ and RNPC1 [30] or p53 itself (Supplementary information, Figure S7B). Furthermore, RoR RNA was pulled down by hnRNP I specific antibody (Figure 4C), further suggesting that RoR interacts with hnRNP I. In addition, hnRNP I also interacted with p53 mRNA, as detected by RNA immunoprecipitation (RIP) (Supplementary information, Figure S7C). As a member of the large hnRNP family [31], hnRNP I has been implicated in mRNA splicing [32]. To demonstrate that hnRNP I is important to p53 induction, we knocked down hnRNP I by RNAi and detected a remarkable reduction of the doxo-induced p53 in the siRNA-treated cells compared to control (Figure 4D). In contrast, ectopic expression of hnRNP I increased the p53 level though at a lesser extent (Figure 4E) presumably because sufficient amount of the endogenous hnRNP I is already in the cell.

RoR interacts with the phosphorylated hnRNP I in the cytoplasm

Although hnRNP I is a nuclear protein, cell fraction- 


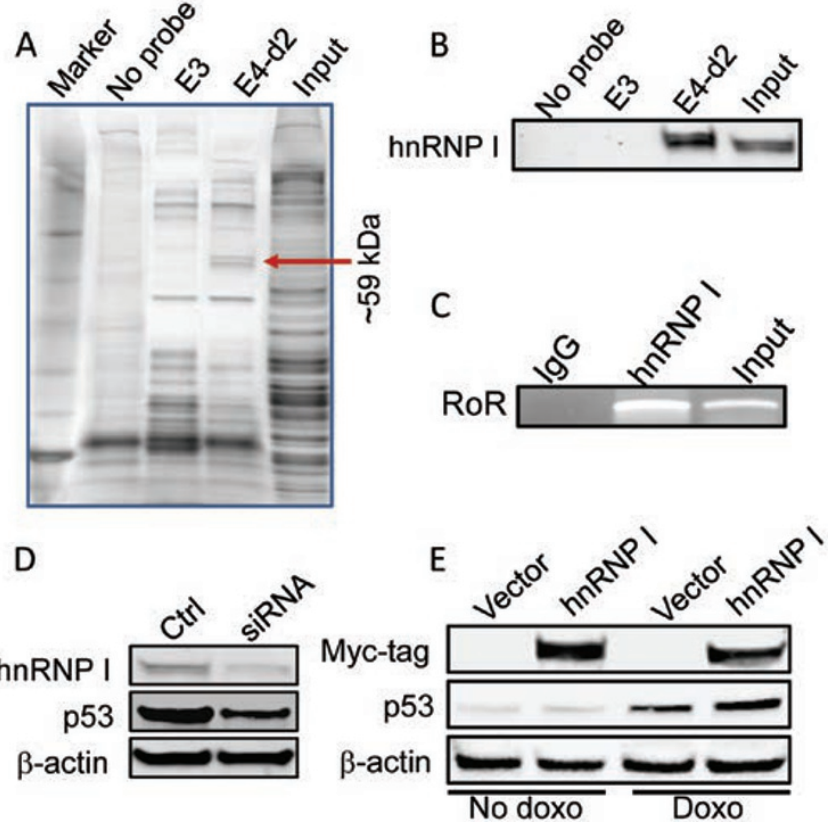

Figure 4 hnRNP I is a RoR binding partner. (A) A representative silver-stained gel picture showing a doublet band unique to E4-d2. Mass spectrometry analysis indicated that this band is hnRNP I. (B) Verification of the interaction of RoR with hnRNP I by RNA precipitation and western blot. Samples were prepared in the same way as in (A) and the membrane was probed with hnRNP I antibody. (C) RT-PCR detection of RoR after the hnRNP I immunoprecipitation using primers ROR-Exon4-RT5.1A and ROR-Exon4-RT-3.1A (Supplementary information, Table S1). (D) Suppression of the doxo-induced p53 by hnRNP I-siRNAs. MCF-7 cells were first transfected with control siRNA or hnRNP-siRNAs (mixed pool), and then treated with $0.5 \mu \mathrm{g} /$ $\mathrm{ml}$ doxo for $16 \mathrm{~h}$ before harvesting cells for western blot. (E) Effect of ectopic expression of hnRNP I on p53. MCF-7 cells were first transfected with vector or hnRNP I, and then treated with $0.2 \mu \mathrm{g} / \mathrm{ml}$ doxo for $16 \mathrm{~h}$ before western blot. The purpose of using a low concentration of doxo was to not allow p53 induction to reach the maximum so that we would be able to detect any further increase in the p53 level by hnRNP I.

ation experiments revealed a small amount of hnRNP I in the cytoplasm (Figure 5A). Of great interest, RNA precipitation combined with western blot suggested that the interaction between hnRNP I and RoR occurs predominantly in the cytoplasm because a strong signal was detected in the cytosolic fraction, whereas little signal was detected in the nuclear fraction (Figure 5B). Furthermore, in situ hybridization (ISH) revealed that RoR is mainly present in the cytoplasm (Supplementary information, Figure S8A). Previous reports indicated that phosphorylation impacts the subcellular localization of hnRNP I [33, 34]. Indeed, immunofluorescence microscopy showed that while a majority of the protein is unphosphorylated and stays in the nucleus, phosphorylated hnRNP I (p-hnRNP I) was present in the cytoplasm (Figure 5C; Supplementary information, Figure S8B). In particular, protein kinase A (PKA) serves a kinase for hnRNP I phosphorylation [33]. Thus, we ectopically expressed PKA and detected an increase in p-hnRNP I (Figure 5D). Consistent with this finding, hnRNP I pulled down by E4-d2 probe was mainly the phosphorylated form, $\mathrm{p}$-hnRNP I; moreover, a higher level of p-hnRNP I was seen in the PKA-transfected cells compared to the vector control cells (Figure $5 \mathrm{E}$ ). To further demonstrate the role of phosphorylation of hnRNP I in its ability to interact with RoR, we made a phosphorylation dead mutant (S16A) [33] (Supplementary information, Figure $\mathrm{S} 8 \mathrm{C}$ ). As expected, the wild type, but not the mutant hnRNP I, was recovered from the RoR RNA precipitates (Figure 5F), thus demonstrating that RoR preferably interacts with p-hnRNP I.

\section{A 28-base RoR sequence carrying hnRNP I binding mo- tifs is fully functional for $p 53$ repression}

To determine the minimal region required for $\mathrm{p} 53$ repression, we made two additional deletions and narrowed down the active region within a $165 \mathrm{bp}$ fragment (E4-d3B) (Supplementary information, Figure S9A). Of considerable interest, we found two potential hnRNP I binding motifs in this region (Supplementary information, Figure S9B) that are very similar to the conserved hnRNP I binding motif [35]. Deletion of these two potential hnRNP I binding motifs (E4-d4) abolished the suppression activity (Figure 5G; Supplementary information, Figure S9B and S9C), suggesting that these two motifs are essential. To further determine their role in p53 repression, we introduced a synthetic RNA oligo (RoR-oligo-1) (Supplementary information, Figure S9C) into the cells, and demonstrated that RoR-oligo-1 alone was sufficient to suppress p53 (Figure 5G). In contrast, the same oligo with mutations at 4 conserved cytosines (RoR-oligo-M) lost its ability to suppress p53 (Figure 5G; Supplementary information, Figure S9C), highlighting the critical role of these 4 cytosines. Furthermore, biotin-labeled RoR-oligo-1 was able to successfully pull down hnRNP I, whereas the same oligo with mutations at 4 conserved cytosines (Biotin-oligo-M) was not (Figure $5 \mathrm{H})$. Therefore, RoR-oligo-1 possesses full function of RoR for $\mathrm{p} 53$ repression. Together, these results suggest that RoR suppresses p53 through its interaction with the cytoplasmic p-hnRNP I at the hnRNP I binding motifs.

\section{p53 transcriptionally induces RoR}

Finally, we showed that RoR itself is under regulation of p53. Doxo induced p53 and at the same time, it 


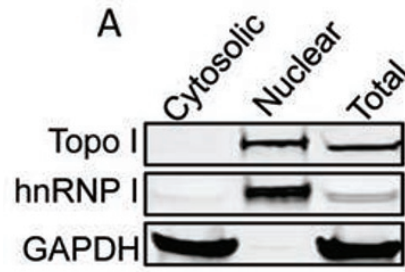

D

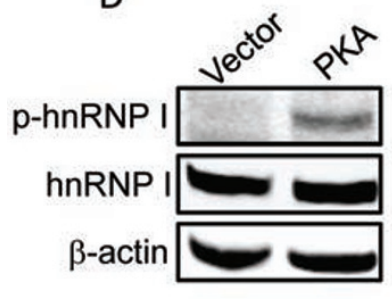

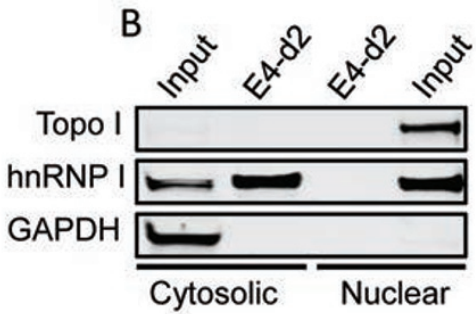

E

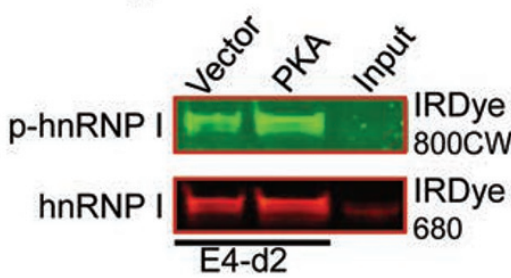

C

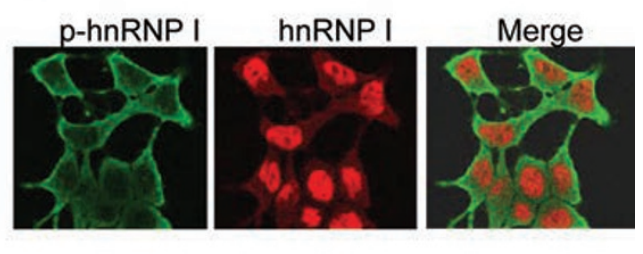

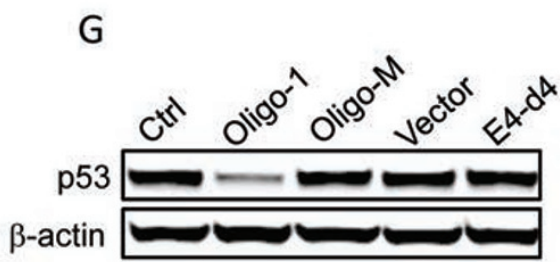

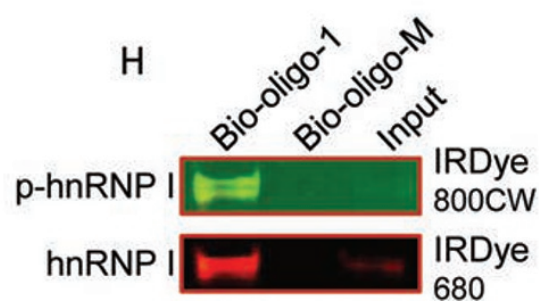

Figure 5 p-hnRNP I is localized to the cytoplasm and interacts with RoR at the hnRNP I binding motifs. (A) Subcellular localization of hnRNP I by cell fractionation. After cell fractionation of MCF-7 cells, an equal amount of protein (30 $\mu \mathrm{g} / \mathrm{lane}) \mathrm{was}$ loaded for each lane. Note that a very weak hnRNP I band was detected in the cytosolic fraction whereas a strong band came from the nuclear fraction. Topoisomerase I (topo I) serves as a nuclear protein marker; GAPDH as a cytosolic protein marker. (B) Detection of hnRNP I protein after RNA precipitation by western blot. The same cytosolic and nuclear extracts from (A) were used for RNA precipitation. However, since over $95 \%$ of hnRNP I is present in the nucleus, we adjusted the amount of protein and used the nuclear protein of about $1 / 5$ amount of the cytosolic protein for RNA precipitation. (C) Detection of hnRNP I and p-hnRNP I by confocal immunofluorescence microscopy. While hnRNP I (red) is predominantly in the nucleus, p-hnRNP I (green) is in the cytoplasm. (D) PKA enhances hnRNP I phosphorylation. (E) RoR-bound hnRNP I is phosphorylated and PKA enhances the interaction of RoR with hnRNP I. Total cellular extract from the same transfected cells in (D) was used for RNA precipitation. The same membrane was probed simultaneously with hnRNP I antibody (rabbit origin) and p-hnRNP I antibody (mouse origin), followed by secondary antibody probing as indicated. (F) Serine phosphorylation of hnRNP I is essential for its interaction with RoR. MCF-7 cells were transfected with wild-type hnRNP I or mutant hnRNP I (S16A). Total cellular extract was prepared for RNA precipitation. (G) Suppression of p53 by RoR-oligo-1. MCF-7 cells were transfected with vector or E4$\mathrm{d} 4$; or control oligo, oligo-1 or oligo-M. The transfected cells were then treated with doxo at $1 \mu \mathrm{g} / \mathrm{ml}$ for $24 \mathrm{~h}$ before protein extraction for western blot. $(\mathrm{H})$ Precipitation of hnRNP I by biotin-labeled RNA oligos (Supplementary information, Figure S9B). Total cellular extract from non-transfected MCF-7 cells was prepared for RNA precipitation. The same membrane was probed simultaneously with hnRNP I antibody (rabbit origin) and p-hnRNP I antibody (mouse origin), followed by secondary antibody probing as indicated.

increased the RoR level, as detected by qRT-PCR (Figure 6A). Moreover, ectopic expression of $\mathrm{p} 53$ also induced RoR (Figure 6A). However, p53 with a point mutation $(\mathrm{R} 175 \mathrm{H})$ at the DNA binding domain, a frequent mutant in cancer [36], had no effect on RoR expression. The p53 scan program [37] identified 4 potential p53 response elements (p53REs) within a $1 \mathrm{~Kb}$ fragment upstream of
RoR (Supplementary information, Figure S10A). It is evident that p53RE-1 is the most conserved among all four p53REs (Supplementary information, Figure S10A). Consistent with qRT-PCR results, luciferase assays with a reporter carrying this $1 \mathrm{~Kb}$ fragment revealed that $\mathrm{p} 53$ increased the luciferase activity by 3 -fold (Figure 6B). Again, the mutant p53 had no effect on the luciferase 
A

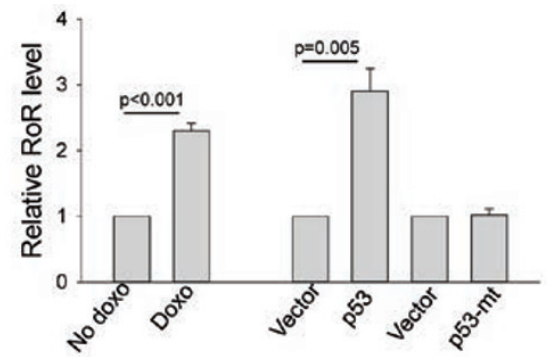

B
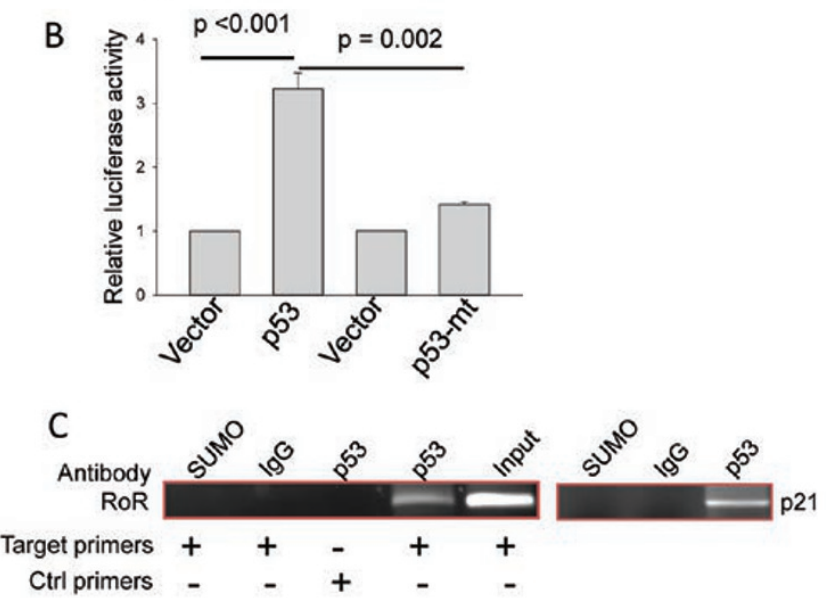

Figure 6 p53 induces RoR by binding to a p53 response element in the RoR promoter. (A) Induction of RoR by p53. Cells were treated with doxo at $1 \mu \mathrm{g} / \mathrm{ml}$ for $24 \mathrm{~h}$ and then RNA was extracted for qRT-PCR. In addition, HCT-116 WT cells were transfected with vector alone or WT-p53 or mutant p53 $(\mathrm{R} 175 \mathrm{H})$. Values are mean \pm SE $(n=3)$. (B) Induction of the RoR promoter luciferase activity by p53. A $1000 \mathrm{bp}$ fragment upstream of RoR was cloned into pGL3-Basic as a luciferase reporter. 293T cells were transfected with the luciferase reporter along with wild-type or mutant $(\mathrm{R} 175 \mathrm{H}) \mathrm{p} 53$. Luciferase assay was performed $24 \mathrm{~h}$ after transfection. Values are mean $\pm \mathrm{SE}(n=3)$. (C) Detection of $p 53$ interaction with $p 53 R E-1$ by ChIP assay. PCR primers (RoR-ChIP-p53-1-5.1 and RoR-ChIP-p53-1-3.1) were indicated by red arrows (see Supplementary information, Figure S10B). SUMO antibody serves as a negative control, and p21 serves as a positive control.

activity (Figure 6B). Deletion and site-directed mutagenesis showed that p53RE-1 was important to the p53induced activity (Supplementary information, Figure S10B). Deletion of all four p53REs or p53RE-1 alone decreased the luciferase activity to below $40 \%$; similarly, the reporter with mutations in p53RE-1 also showed about $40 \%$ of the full-length promoter activity. Finally, chromatin immunoprecipitation (ChIP) assays confirmed that p53 specifically interacted with p53RE-1 (Figure $6 \mathrm{C})$. Therefore, like the negative regulator MDM2, RoR is also under control of p53, forming an autoregulatory feedback loop (Supplementary information, Figure S11), through which p53 may be delicately kept in check so that the cell is able to better respond to intracellular or extracellular stresses.

\section{Discussion}

Given the importance of p53 in diverse cellular pathways, the cellular level of p53 protein is under strict control. To achieve this, the cell may develop a sophisticated regulatory system because an unwanted induction of p53 could be harmful to the cell. For instance, the cell may express a number of negative regulators $[25,26$, 38]. Among them, MDM2 is a major E3 ubiquitin ligase controlling the p53 stability through the ubiquitin-proteasome pathway while allowing its rapid increase in response to stress [21, 39]. However, it is unclear whether there is an additional mechanism that can keep the stressinduced p53 level under control. This study demonstrates that RoR is a unique member of $\mathrm{p} 53$ negative regulators because RoR is capable of suppressing the cellular p53 level after DNA damage; this RoR-mediated p53 repression is in part through translation regulation.

Our study identifies another important protein, hnRNP I, in this RoR-mediated p53 repression network because the ability of RoR to repress p53 is dependent on the interaction of RoR with hnRNP I and suppression of hnRNP I by RNAi substantially decreases the doxoinduced p53, as RoR does. In particular, a 28-base RoR sequence carrying the potential hnRNP I binding motifs is essential and sufficient for this repression, providing further evidence of the involvement of hnRNP I in RoRmediated p53 repression. hnRNP I is an RNA binding protein that carries several RNA binding domains and is well known for its role in mRNA splicing [40]; it is relatively abundant in the cell. While the majority of hnRNP I is retained in the nucleus; small fraction is in the cytoplasm (Figure 5A and 5C). Since mRNA splicing takes place in the nucleus, this function is mostly likely dependent on the nuclear hnRNP I. On the other hand, our study shows that the cytoplasmic hnRNP I is phosphorylated and is responsible for the interaction with RoR because almost all of the pulled down hnRNP I is phosphorylated.

Previous studies suggest that the 5'-UTR of p53 mRNA may form a secondary structure to serve as an internal ribosome entry site (IRES) [41, 42]. Translation regulation can also occur through the 3 '-UTR. For instance, HuR was shown to bind to either the AU-rich or the U-rich regions of the p53 3'-UTR, leading to an increase in p53 translation after UVC treatment [43]. On the other hand, RNPC1 functions as a translational repressor by binding to the $5^{\prime}$ - and $3^{\prime}$-UTR of p 53 mRNA 
so that it prevents cap-binding protein eIF4E from binding to $\mathrm{p} 53$ mRNA [30]. Moreover, $\mathrm{p} 53$ protein is capable of binding to the 5'-UTR of p53 mRNA and inhibiting its translation [44]. We showed that hnRNP I binds to p53 mRNA (Supplementary information, Figure S7C), which is consistent with the report that hnRNP I is capable of binding to the IRES to stimulate p53 translation after DNA damage, leading to the production of the N-terminal truncated isoform of p53 [45]. Thus it is possible that RoR may prevent or interrupt the interaction of hnRNP I with p53 mRNA to suppress p53.

MDM2 is an E3 ubiquitin ligase and regulates the p53 stability; it is no longer able to bind to p53 upon stress, so that p53 is rapidly increased. Unlike MDM2, RoR is able to keep the p53 level low even after DNA damage. This is critical because in this way, RoR may serve as a check point to prevent p53 induction from "out of control". A delicate balance of these two mechanisms would assure cells to precisely respond to various stresses. In addition to negative regulators of $\mathrm{E} 3$ ubiquitin ligases, other notable p53 regulators include ribosomal proteins, such as RPL26 and RPL11, and RNA binding proteins such as RNPC1. For instance, RPL26 has been shown to directly bind to the $5^{\prime}$ - and $3^{\prime}$-UTR of p53 mRNA in a way that facilitates p53 translation, [28, 29] although RPL26 can also interact with MDM2 and regulate the p53 stability [46]. Similarly, ribosomal proteins such as RPL11 have been reported to bind to MDM2 protein and increase the $\mathrm{p} 53$ protein stability by inhibiting the association of MDM2 with p53 [47-49]. Suppression of RPL11 by PICT1 causes p53 repression and promotes cell growth [50]. Although both RPL26 and RNPC1 are involved in p53 translation, we found no evidence that RoR interacts with RPL26 or RNPC1. Thus, the mechanism of $\mathrm{p} 53$ regulation involving these proteins may also be distinct from what we have reported here. Nevertheless, both positive and negative translational control of p53 by these proteins, along with RoR, may be part of the highly coordinated response of cells to DNA damage and other cellular stresses.

One of the interesting findings is the transcriptional regulation of RoR by p53. MDM2 inhibits p53, which, however, is able to induce MDM2. Like MDM2, RoR is also under control of $\mathrm{p} 53$. This autoregulatory feedback loop may signify the critical role of RoR as an important p53 repressor. As discussed above, an unwanted induction of p53 could be deleterious to the cell. In order for the cell to keep p53 at a certain level, the cell has to control the p53 level precisely. One of such mechanisms may involve RoR-mediated suppression because the RoR level is also increased when p53 is induced (Supplementary information, Figure S11). Although the mouse lincR-
NA-p21 [51] and the human lncRNA PANDA [12] were recently shown to be targets of p53, unlike these lncRNAs, RoR functions to suppress $\mathrm{p} 53$ by a novel negative feedback loop. Given that microRNAs are well known as direct p53 targets [52], as a member of lncRNAs, RoR now also joins the p53 network, providing another piece in the tumor suppression puzzle. Along with these findings, our study provides further evidence that non-coding RNAs, particularly RoR in this case, are important interplayers in the p53-mediated tumor suppression network.

\section{Materials and Methods}

\section{Reagents}

Primary antibodies were purchased from the following commercial vendors: p53 (both N- and C-terminal), phosphorylated p53 (Ser15), MDM2 from Epitomics; acetylated p53 (K382), p21, RPL26 from Cell Signaling; hnRNP I, Pirh2 and MDMX from Santa Cruz; COP1 from Abcam; GAPDH from ProteinTech; RNPC1 (or RBM38), $\beta$-actin from Sigma; Myc-tag from Applied Biological Materials (Vancouver, British Columbia, Canada). Secondary antibodies conjugated with IRDye $800 \mathrm{CW}$ or IRDye 680 were from LI-COR Biosciences (Lincoln, NE, USA); ${ }^{35} \mathrm{~S}$ methionine from Perkin Elmer; PCR primers and RNA oligos were purchased from IDT (Coralville, IA, USA); hnRNP I siRNAs (mixed pool) from Santa Cruz. Breast tumor RT-PCR arrays from Origene (Rockville, MD, USA); RoR-siRNA-1/2 and negative control oligo from Thermo Scientific; RoR-LNA oligo for ISH from Exiqon (2950 Vedbaek, Denmark); the PKA cDNA clone from Open Biosystems; mutant p53 (R175H) from Addgene; nutlin from Sigma. The original RoR in pBabe was a generous gift from Dr Daley (Harvard Medical School) and it was used as a PCR template to generate various RoR constructs in this study. Antibody against phnRNP I (Ser16) was a generous gift from Dr Black (UCLA).

\section{Cell culture}

Breast cancer MCF-7 cells (both from ATCC) and colon cancer cell lines HCT-116 (wild-type p53 and p53 null) (provided by Dr. Voglestein at The Johns Hopkins University) were grown in RPMI 1640 (Lonza, Walkersville, MD). HEK-293T (ATCC) cells were cultured in DMEM (Lonza). All culture media were supplemented with $10 \%$ FBS, $2 \mathrm{mM}$ glutamine, 100 units of penicillin/ $/ \mathrm{ml}$, and $100 \mu \mathrm{g}$ of streptomycin $/ \mathrm{ml}$. Cells were incubated at $37{ }^{\circ} \mathrm{C}$ and supplemented with $5 \% \mathrm{CO}_{2}$ in the humidified chamber.

\section{Transfection}

Cells were transfected with siRNAs or RNA oligos using RNAfectin reagent or with plasmid DNA using DNAfectin (Applied Biological Materials) following the manufacturer's protocol. Sequences for RoR-siRNA-1 and RoR-siRNA-2 were described previously [19].

\section{Plasmid construction}

All PCR primers for cloning were listed in Supplementary information, Table S1 and high fidelity enzyme Phusion was used for PCR amplification. The entire RoR sequence was PCR amplified from the original RoR expression vector in $\mathrm{pBabe}$ using primers RoR-R1-5.1 (sense) and RoR-Not1-3.1 (antisense) and then 
directly cloned into a lentiviral vector under MSCV promoter by Cold Fusion kit (SBI). The same strategy was used to clone various lengths of RoR fragments in this study unless it was specifically stated. The cloning of RoR putative promoter used human genomic DNA as a template with RoRp-Xho1-5.1 and RoRpXho1-3.1 and was cloned into pGL3-basic (Promega) at Xho I site by Cold Fusion; various deletions and site-directed mutagenized sequences were also made in the same vector. PKA, hnRNP1, RPL26 and RNPC1 were cloned into a modified pCDH-CMVEF1-copGFP that carried Myc-tag at the N-terminus. RoR was also cloned into a mCherry-carrying expression vector by a standard ligation method; this clone was used in TUNEL assays. To make RoR or RoR promoter constructs carrying deletions or mutations, a two-step PCR procedure was used as described previously [53]. All PCR products were verified by DNA sequencing.

\section{Luciferase assay}

Luciferase assays were performed using a luciferase assay kit (Promega) according to the manufacturer's protocol. Briefly, cells were first transfected with appropriate plasmids in 12-well plates. Then the cells were harvested and lysed for luciferase assay $24 \mathrm{~h}$ after transfection. Renilla luciferase was used for normalization.

\section{$q R T-P C R$}

To detect the transcriptional level of RoR and p53, we used the SYBR Green method with primers listed in Supplementary information, Table S1. PCR was carried out in ABI 7500 real time PCR system. PCR results, recorded as threshold cycle numbers $(\mathrm{Ct})$, were normalized against an internal control ( $\beta$-actin).

\section{Western blot}

Cells were harvested and protein was extracted from transfected cells as previously described [20] using pre-casted SDS-gels (Thermo Scientific) at $12 \%$ or $4 \%-20 \%$ gradient.

\section{Immunofluorescence microscopy}

To determine the subcellular localization of hnRNP I, we performed immunofluorescence staining using the hnRNP I as well as p-hnRNP I antibody according to the manufacturer's protocol (Invitrogen). The signal was revealed by a secondary antibody conjugated with Alexa Fluor 488 or Alexa Fluor 568. Confocal microscopy was carried out by using the Leica confocal microscope at the imaging facility of Southern Illinois University School of Medicine.

\section{TUNEL assays}

MCF-7 cells were transfected with vector or RoR carrying mCherry and then treated with doxo at $2 \mu \mathrm{g} / \mathrm{ml}$ for $24 \mathrm{~h}$. After washing twice with PBS, the cells were fixed with $4 \%$ paraformaldehyde for $15 \mathrm{~min}$ at room temperature, and permeabilized in $0.25 \%$ Triton-X 100 for $20 \mathrm{~min}$ at room temperature. TUNEL assays were performed according to the manufacturer's instructions (Invitrogen). Briefly, the cells were first incubated in TdT reaction cocktail for $60 \mathrm{~min}$ at $37^{\circ} \mathrm{C}$, followed by treatment with Click-iT reaction cocktail. The nucleus was stained with Hoechst 33342.

\section{Cell cycle analysis}

Cell cycle analysis was performed using the standard propidium iodide method. In brief, HCT-116 WT, HCT-116 p53-null cells were first transfected with either vector control or RoR expression vector. The transfected cells were then treated with doxo at $0.1 \mu \mathrm{g} /$ $\mathrm{ml}$ for $16 \mathrm{~h}$ before harvesting for cell cycle analysis. After fixing with $70 \%$ ethanol, the cells were stained with propidium iodide along with RNase A. Finally, the cells were analyzed by FACS Vantage flow cytometer (Becton-Dickinson) at the core facility of Southern Illinois University School of Medicine.

\section{RIP}

To determine whether hnRNP I interacts with RoR, we used hnRNP I antibody to pull down hnRNP I and then detected RoR RNA by RT-PCR using RoR specific primers (ROR-Exon4-RT5.1A and ROR-Exon4-RT-3.1A) in Supplementary information, Table S1. Magna RIPTM RNA-Binding Protein Immunoprecipitation Kit (Millipore) was used for RIP procedures according to the manufacturer's protocol. After the antibody was recovered by protein $\mathrm{A}+\mathrm{G}$ beads, standard RT-PCR was performed to detect RoR RNA in the precipitates.

\section{$R N A$ precipitation}

This experiment used RNA probes to precipitate RNA binding proteins. Thus, the DNA fragment covering RoR exon 3 or E4-d2 was PCR-amplified using a T7 containing primer and then cloned into pCR8 (Invitrogen). In addition, the full-length RoR was also cloned and used in precipitation experiments for comparison. The resultant plasmid DNA was linearized with restriction enzyme Not I (the stie was introduced from the reverse PCR primer), and then used to synthesize RNA by T7 polymerase. A $20 \mu \mathrm{l}$ reaction contained $400 \mathrm{ng}$ linearized plasmid DNA, $20 \mathrm{U}$ ribonuclease inhibitor, $2.5 \mathrm{mM}$ NTP mixture supplemented with $10 \%$ biotinlabeled UTP (Perkin Elmer) and 20 U T7 RNA polymerase (New England BioLabs); and then it was incubated at $37{ }^{\circ} \mathrm{C}$ for $60 \mathrm{~min}$, followed by 25 U RNase-free DNase I (New England BioLabs) treatment at $37^{\circ} \mathrm{C}$ for $30 \mathrm{~min}$. The labeled RNA was purified by a column-based kit (Zymo Research). Cellular extract was prepared from a $10 \mathrm{~cm}$ dish culture with $\sim 80 \%$ confluence (about 8 million cells) with protein lysis buffer [54]. In addition to total protein extract, we also prepared cytosolic and nuclear fractions with cell membrane lysis buffer (50 mM KCl, 25 mM HEPES, pH 7.8, 100 $\mu \mathrm{M}$ DTT, $0.5 \% \mathrm{NP}-40$ ) containing protease inhibitor cocktails (Sigma). After a brief spin, supernatant was saved as a cytosolic fraction, and nuclear lysis buffer $\left(500 \mathrm{mM} \mathrm{KCl}, 1.5 \mathrm{mM} \mathrm{MgCl}_{2}\right.$, 25 mM HEPES, pH 7.8, $100 \mu \mathrm{M}$ DTT, 10\% glycerol) containing protease inhibitor cocktails was then added to the pellet to extract nuclear protein. For precipitation assays, the reaction (RNA probe and protein extract) was incubated at $4{ }^{\circ} \mathrm{C}$ for $60 \mathrm{~min}$, followed by 5 washes with PBS. For experiments with biotin-labeled RoRoligo-1 and mutant oligo-1, the RNA oligos (100 pmole each) were directly used in precipitation assay.

\section{Pulse-chase experiment}

MCF-7 cells were first transfected with control siRNA or RoRsiRNA-2 overnight and then grown in regular DMEM medium at the exponential stage before replacing pre-warm DMEM minus methionine with 10\% dialyzed FBS (Invitrogen) and incubated for $30 \mathrm{~min}$. After removal of medium, the cells were incubated in the same fresh medium containing $30 \mu \mathrm{l}$ of $\sim 10 \mu \mathrm{Ci} / \mu \mathrm{L} \mathrm{L}-{ }^{35} \mathrm{~S}$ methionine (Perkin Elmer) for $1 \mathrm{~h}$. Finally, the radioactive medium was removed and fresh warm DMEM $+10 \%$ FBS $+2 \mathrm{mM}$ cold methi- 
onine was added to dishes. After incubation for $30 \mathrm{~min}$, the cells were harvested for IP with anti-p53 antibody, followed by SDSPAGE. The gel was dried under vacuum, and then exposed to an $\mathrm{X}$-ray film for 16-24 $\mathrm{h}$ before development.

\section{Protein ID identification by mass spectrometry}

After RNA precipitation, samples were separated in SDSPAGE, followed by silver staining (Pierce) according to the manufacturer's protocol. Candidate protein bands were carefully cut out and sent out for mass spectrometry analysis provided by Applied Biomics.

\section{ChIP}

ChIP assays were performed using a commercial kit from Cell Signaling. Briefly, cells were first fixed with formaldehyde, and chromatin DNA was isolated and digested with nucleases. PCR was performed using primers RoR-ChIP-p53-1-5.1 and RoRChIP-p53-1-3.1 or control primers RoR-ChIP-p53-1-5.1A and RoR-ChIP-p53-1-3.1A (Supplementary information, Table S1). $\mathrm{IgG}$ and an unrelated antibody (anti-SUMO) were used as negative controls; p21 was used as a positive control.

\section{ISH}

ISH was used to detect RoR expression in cultured MCF-7 cells using a biotin-labeled antisense RoR-LNA probe according to a published method [55]. In brief, cells were first grown on coverslips overnight and then fixed with $1 \%$ paraformaldehyde. Following pre- and hybridization, and washes, the signal was amplified by TSA amplification kit (Perkin Elmer) and subsequently revealed by Ultra Vision One polymer and AEC chromogen (Thermo Scientific).

\section{Statistical analysis}

Data are presented as mean $\pm \mathrm{SE}$; the Student's $t$-test was used for assessing the difference between individual groups and $P \leq 0.05$ was considered statistically significant.

\section{Acknowledgments}

We are grateful to Dr George Daley (Harvard Medical School) for providing the original clone of RoR; Dr Douglas Black (University of California, Los Angeles) for phosphorylated hnRNP I antibody, and Dr Vert Vogelstein (Johns Hopkins University) for HCT-116 p53 WT and HCT-116 p53 null cells. We thank Dr Xiaowei Wang (Washington University at St. Louis) for helping us with UCSC genome browser analysis. This work was supported by NIH grants R01 CA154989 (YM) and R01 CA124650 (KW).

\section{References}

1 Levine AJ, Oren M. The first 30 years of $\mathrm{p} 53$ : growing ever more complex. Nat Rev Cancer 2009; 9:749-758.

2 Vousden KH, Prives C. Blinded by the light: the growing complexity of p53. Cell 2009; 137:413-431.

3 Meek DW, Anderson CW. Posttranslational modification of p53: cooperative integrators of function. Cold Spring Harb Perspect Biol 2009; 1:a000950.

4 Halaby MJ, Yang DQ. p53 translational control: a new facet of p53 regulation and its implication for tumorigenesis and cancer therapeutics. Gene 2007; 395:1-7.

5 Birney E, Stamatoyannopoulos JA, Dutta A, et al. Identification and analysis of functional elements in $1 \%$ of the human genome by the ENCODE pilot project. Nature 2007; 447:799816.

6 Prensner JR, Chinnaiyan AM. The emergence of lncRNAs in cancer biology. Cancer Discov 2011; 1:391-407.

7 He Y, Vogelstein B, Velculescu VE, Papadopoulos N, Kinzler KW. The antisense transcriptomes of human cells. Science 2008; 322:1855-1857.

8 Swami M. Small RNAs: Pseudogenes act as microRNA decoys. Nat Rev Cancer 2010; 10:535.

9 Poliseno L, Salmena L, Zhang J, Carver B, Haveman WJ, Pandolfi PP. A coding-independent function of gene and pseudogene mRNAs regulates tumour biology. Nature 2010; 465:1033-1038.

10 Gupta RA, Shah N, Wang KC, et al. Long non-coding RNA HOTAIR reprograms chromatin state to promote cancer metastasis. Nature 2010; 464:1071-1076.

11 Guttman M, Donaghey J, Carey BW, et al. lincRNAs act in the circuitry controlling pluripotency and differentiation. $\mathrm{Na}$ ture 2011; 477:295-300.

12 Hung T, Wang Y, Lin MF, et al. Extensive and coordinated transcription of noncoding RNAs within cell-cycle promoters. Nat Genet 2011; 43:621-629.

13 Khalil AM, Guttman M, Huarte M, et al. Many human large intergenic noncoding RNAs associate with chromatin-modifying complexes and affect gene expression. Proc Natl Acad Sci USA 2009; 106:11667-11672.

14 Prensner JR, Iyer MK, Balbin OA, et al. Transcriptome sequencing across a prostate cancer cohort identifies PCAT-1, an unannotated lincRNA implicated in disease progression. Nat Biotechnol 2011; 29:742-749.

15 Tsai MC, Manor O, Wan Y, et al. Long noncoding RNA as modular scaffold of histone modification complexes. Science 2010; 329:689-693.

16 Wang KC, Yang YW, Liu B, et al. A long noncoding RNA maintains active chromatin to coordinate homeotic gene expression. Nature 2011; 472:120-124.

17 Mercer TR, Dinger ME, Mattick JS. Long non-coding RNAs: insights into functions. Nat Rev Genet 2009; 10:155-159.

18 Wang KC, Chang HY. Molecular mechanisms of long noncoding RNAs. Mol Cell 2011; 43:904-914.

19 Loewer S, Cabili MN, Guttman M, et al. Large intergenic non-coding RNA-RoR modulates reprogramming of human induced pluripotent stem cells. Nat Genet 2010; 42:11131117.

20 Sachdeva M, Zhu S, Wu F, et al. p53 represses c-Myc through induction of the tumor suppressor miR-145. Proc Natl Acad Sci USA 2009; 106:3207-3212.

21 Kruse JP, Gu W. Modes of p53 regulation. Cell 2009; 137:609-622.

22 Sakaguchi K, Herrera JE, Saito S, et al. DNA damage activates p53 through a phosphorylation-acetylation cascade. Genes Dev 1998; 12:2831-2841.

23 Vassilev LT, Vu BT, Graves B, et al. In vivo activation of the p53 pathway by small-molecule antagonists of MDM2. Science 2004; 303:844-848.

24 Torarinsson E, Yao Z, Wiklund ED, et al. Comparative ge- 
nomics beyond sequence-based alignments: RNA structures in the ENCODE regions. Genome Res 2008; 18:242-251.

25 Shvarts A, Steegenga WT, Riteco N, et al. MDMX: a novel p53-binding protein with some functional properties of MDM2. EMBO J 1996; 15:5349-5357.

26 Dornan D, Wertz I, Shimizu H, et al. The ubiquitin ligase COP1 is a critical negative regulator of p53. Nature 2004; 429:86-92.

27 Leng RP, Lin Y, Ma W, et al. Pirh2, a p53-induced ubiquitinprotein ligase, promotes p53 degradation. Cell 2003; 112:779791.

28 Chen J, Kastan MB. 5'-3'-UTR interactions regulate p53 mRNA translation and provide a target for modulating p53 induction after DNA damage. Genes Dev 2010; 24:2146-2156.

29 Takagi M, Absalon MJ, McLure KG, Kastan MB. Regulation of p53 translation and induction after DNA damage by ribosomal protein L26 and nucleolin. Cell 2005; 123:49-63.

30 Zhang J, Cho SJ, Shu L, et al. Translational repression of $\mathrm{p} 53$ by RNPC1, a p53 target overexpressed in lymphomas. Genes Dev 2011; 25:1528-1543.

31 Dreyfuss G, Matunis MJ, Pinol-Roma S, Burd CG. hnRNP proteins and the biogenesis of mRNA. Annu Rev Biochem 1993; 62:289-321.

32 Izquierdo JM, Majos N, Bonnal S, et al. Regulation of Fas alternative splicing by antagonistic effects of TIA-1 and PTB on exon definition. Mol Cell 2005; 19:475-484.

33 Xie J, Lee JA, Kress TL, Mowry KL, Black DL. Protein kinase A phosphorylation modulates transport of the polypyrimidine tract-binding protein. Proc Natl Acad Sci USA 2003; 100:8776-8781.

34 Knoch KP, Meisterfeld R, Kersting S, et al. cAMP-dependent phosphorylation of PTB1 promotes the expression of insulin secretory granule proteins in beta cells. Cell Metab 2006; 3:123-134.

35 Tillmar L, Carlsson C, Welsh N. Control of insulin mRNA stability in rat pancreatic islets. Regulatory role of a 3'-untranslated region pyrimidine-rich sequence. $J$ Biol Chem 2002; 277:1099-1106.

36 Soussi T, Ishioka C, Claustres M, Beroud C. Locus-specific mutation databases: pitfalls and good practice based on the p53 experience. Nat Rev Cancer 2006; 6:83-90.

37 Smeenk L, van Heeringen SJ, Koeppel M, et al. Characterization of genome-wide p53-binding sites upon stress response. Nucleic Acids Res 2008; 36:3639-3654.

38 Carvajal D, Tovar C, Yang H, Vu BT, Heimbrook DC, Vassilev LT. Activation of p53 by MDM2 antagonists can protect proliferating cells from mitotic inhibitors. Cancer Res 2005; 65:1918-1924.

39 Meek DW. Tumour suppression by p53: a role for the DNA damage response? Nat Rev Cancer 2009; 9:714-723.

40 Oberstrass FC, Auweter SD, Erat M, et al. Structure of PTB bound to RNA: specific binding and implications for splicing regulation. Science 2005; 309:2054-2057.

41 Ray PS, Grover R, Das S. Two internal ribosome entry sites mediate the translation of p53 isoforms. EMBO Rep 2006;
7:404-410.

42 Yang DQ, Halaby MJ, Zhang Y. The identification of an internal ribosomal entry site in the $5^{\prime}$-untranslated region of p53 mRNA provides a novel mechanism for the regulation of its translation following DNA damage. Oncogene 2006; 25:4613-4619.

43 Mazan-Mamczarz K, Galban S, Lopez de Silanes I, et al. RNA-binding protein HuR enhances p53 translation in response to ultraviolet light irradiation. Proc Natl Acad Sci USA 2003; 100:8354-8359.

44 Mosner J, Mummenbrauer T, Bauer C, Sczakiel G, Grosse F, Deppert W. Negative feedback regulation of wild-type p53 biosynthesis. EMBO J 1995; 14:4442-4449.

45 Grover R, Ray PS, Das S. Polypyrimidine tract binding protein regulates IRES-mediated translation of p53 isoforms. Cell Cycle 2008; 7:2189-2198.

46 Ofir-Rosenfeld Y, Boggs K, Michael D, Kastan MB, Oren M. Mdm2 regulates p53 mRNA translation through inhibitory interactions with ribosomal protein L26. Mol Cell 2008; 32:180-189.

47 Lohrum MA, Ludwig RL, Kubbutat MH, Hanlon M, Vousden KH. Regulation of HDM2 activity by the ribosomal protein L11. Cancer Cell 2003; 3:577-587.

48 Bhat KP, Itahana K, Jin A, Zhang Y. Essential role of ribosomal protein L11 in mediating growth inhibition-induced p53 activation. EMBO J 2004; 23:2402-2412.

49 Zhang Y, Lu H. Signaling to p53: ribosomal proteins find their way. Cancer Cell 2009; 16:369-377.

50 Sasaki M, Kawahara K, Nishio M, et al. Regulation of the MDM2-P53 pathway and tumor growth by PICT1 via nucleolar RPL11. Nat Med 2011; 17:944-951.

51 Huarte M, Guttman M, Feldser D, et al. A large intergenic noncoding RNA induced by p53 mediates global gene repression in the p53 response. Cell 2010; 142:409-419.

52 He L, He X, Lowe SW, Hannon GJ. microRNAs join the p53 network--another piece in the tumour-suppression puzzle. Nat Rev Cancer 2007; 7:819-822.

53 Zhu S, Si ML, Wu H, Mo YY. MicroRNA-21 targets the tumor suppressor gene tropomyosin 1 (TPM1). J Biol Chem 2007; 282:14328-14336.

54 Wu F, Chiocca S, Beck WT, Mo YY. Gam1-associated alterations of drug responsiveness through activation of apoptosis. Mol Cancer Ther 2007; 6:1823-1830.

55 Gupta A, Mo YY. Detection of microRNAs in cultured cells and paraffin-embedded tissue specimens by in situ hybridization. Methods Mol Biol 2011; 676:73-83.

(Supplementary information is linked to the online version of the paper on the Cell Research website.)

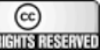

This work is licensed under the Creative Commons Attribution-NonCommercial-No Derivative Works 3.0 Unported License. To view a copy of this license, visit http:// creativecommons.org/licenses/by-nc-nd/3.0 